\title{
$\begin{array}{ll}\text { Research Square } & \begin{array}{l}\text { Preprints are preliminary reports that have not undergone peer review. } \\ \text { They should not be considered conclusive, used to inform clinical practice, } \\ \text { or referenced by the media as validated information. }\end{array}\end{array}$
}

\section{Do different doses of acupuncture matter on autonomic nervous activity and symptom management in dysmenorrhea? A randomized controlled trial.}

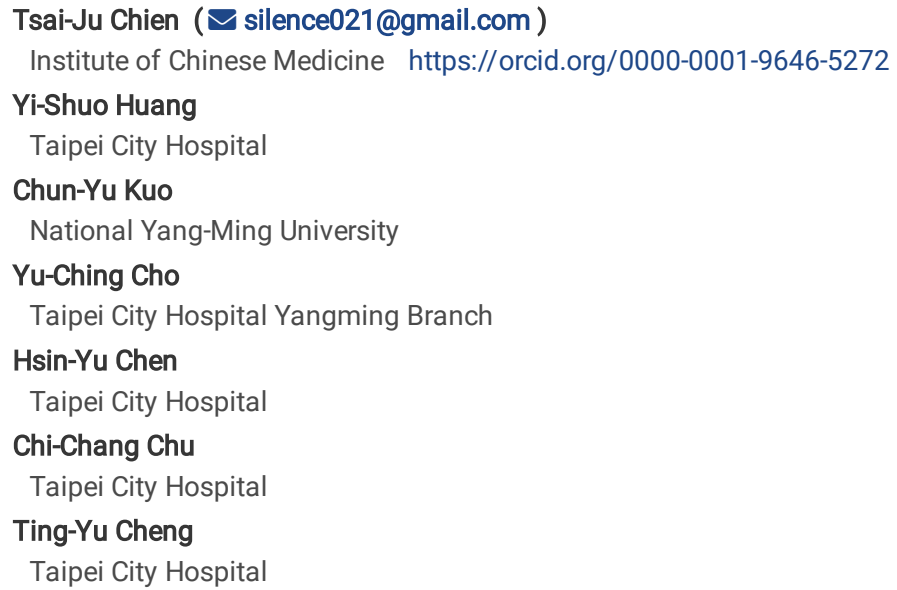




\section{Abstract}

Background The study examined whether the acupuncture dose (number of acupoints stimulated) impacted the efficacy of acupuncture on dysmenorrhea and the relationship with autonomic nervous system regulation.

Methods This three-arm randomized controlled study included a high-dose acupuncture (12 acupoints, $\mathrm{N}=23$ ), low-dose acupuncture (6 acupoints, $\mathrm{N}=30$ ) and control $(\mathrm{N}=30)$ arm. The treatment course was three months. We set heart rate variation (HRV) and analgesics dependence as the primary outcome measurements; Visual Analog Scale (VAS) score, Verbal Multidimensional Scoring System (VMSS) and the 12-Item Short Form Health Survey (SF-12) quality of life questionnaires were set as secondary outcomes. SPSS version 24 was used for data analysis.

Results Low-dose acupuncture was superior to high-dose in analgesics dependence ( $p$ value: low $/$ high $/$ control: $p=0.043 / p=0.056 / p=0.376$ ); symptom relief (VMSS: low/high/control: $p<0.001 / p=0.007 / p=0.109$ ); and physical quality of life (low/high/control: $p<0.001 / p=0.01 / p=0.007$ ). The groups did not differ in HRV parameters $(p>0.05)$. In intergroup analysis, more significant changes were noted in the high-dose than in the low-dose group. The scattered nature of the significant changes implies that acupuncture may have a short-term effect on HRV parameters which does not correlate with the acupuncture dose.

Conclusions Acupuncture can effectively treat dysmenorrheal pain, improve symptoms and reduce analgesic dependence, but the effect does not correlate with the number of acupoints stimulated. The acupuncture has short-term effect on HRV; yet whether its efficacy on dysmenorrhea is directly related to adjusting the autonomic nervous system may need more large-scale study. It is a safe and effective alternative therapy for dysmenorrhea.

Trial: The Efficacy of Different Doses of Acupuncture in Dysmenorrhea, NCT03881319 at ClinicalTrials.gov,

\section{Background}

Dysmenorrhea is a sort of period pelvic pain caused by an abrupt decrease in blood flow and ischemia due to frequent contraction of the uterus. Many females are afflicted with dysmenorrhea to different extents. Some suffer from depression and reduced quality of life while others may even need to withdraw from work or school because of their impaired ability to perform daily activities(1). Thus, dysmenorrhea represents a huge, but hidden, health burden and is worthy of our attention.

The prevalence of dysmenorrhea varies from 34-94\%(2). Generally speaking, primary dysmenorrhea is menstrual pain in the absence of pelvic pathology, while secondary dysmenorrhea is caused by organic dysfunction such as endometriosis, uterus malformation, cervix structure, pelvic inflammation, or other conditions(3). In clinical practice, gynecologists apply analgesics such as Non-Steroidal Anti-Inflammatory Drugs (NSAIDs) and oral contraceptive pills (OCT) or progestin as conventional therapy(4). More than two-thirds of women with dysmenorrhea take analgesics(5) and nearly $80 \%$ have adopted some complementary therapy, such as herbal medicine, acupuncture, moxibustion or aromatherapy. As NSAIDs may cause gastrointestinal discomfort or dyspepsia and hormone therapy can lead to other concerns, some women now adopt acupuncture as a safer therapeutic alternative(6, 7). Women who adopt acupuncture as an alternative therapy usually wish to withdraw the medication use or improve their pain and other symptoms in order improve their quality of life.(8) As acupuncture has been widely used in dysmenorrhea, we observe that people expect greater efficacy with the use of more needles of stimulation; however, the relationship of acupoint selection and optimal treatment is not established(9). No study has yet compared the efficacy of different schedules of acupoints in treating dysmenorrhea, and the acupoints used differ in different trials. We therefore wanted to evaluate whether the number (or dose) of acupoints would affect the outcomes of acupuncture in dysmenorrhea.

Furthermore, in terms of the mechanism of acupuncture, the symptoms related to dysmenorrhea such as anxiety, insomnia and diarrhea are non-specific and related to autonomic dysfunction, namely, vago-sympathetic tone imbalance(10). A previous study also noted that women with dysmenorrhea had decrease heart rate variation (HRV) and autonomic dysfunction compared with the control group(11). Research confirmed that dysmenorrhea afflicted pain and lowered high frequency (HF), indicting lower parasympathetic tone and increasing sympathetic tone(12), compatible with disharmony in the yin/yang. As acupuncture has proven efficacy in adjusting autonomic dysfunction $(13,14)$ and treating dysmenorrhea( $(7,15)$, we sought to understand whether the effect of acupuncture in dysmenorrhea was related to adjustments in the autonomic nervous system. Lastly, we wanted to know if acupuncture could help women with dysmenorrhea take fewer NSAIDs or improve their quality of life.

According to the theory of Traditional Chinese Medicine, acupuncture regulates the energy of the meridians and coordinates the balance of yin/yang(16, 17). Western medicine interprets the mechanism of pain control of acupuncture as an effect of neurophysiology(18) and the release of neuropeptides such as endorphin(19). Additionally, in our previous study, we noted that the theory of yin/yang (meridian) is compatible with the autonomic nerve theory(20). Therefore, in this clinical trial, we aim to evaluate: 1) the effect of different acupuncture doses (by number of acupoints) in dysmenorrhea; 2) the relationship of the mechanism of acupuncture with the adjustment of autonomic nerve activity in dysmenorrhea; and 3) the effect of acupuncture on analgesic dependence in women with dysmenorrhea.

\section{Methods}

\section{Subjects and Allocation}

We selected the participants according to the inclusion criteria: 1) a diagnosis of primary dysmenorrhea according to the Primary Dysmenorrhea Consensus Guideline (21); 2) age from 16 to 35 years without history of delivery; 3 ) normal menstrual cycle (28 \pm 7 days and duration $7 \pm 3$ days); and 4 ) menstrual pain scoring more than 40 on a 100-point Visual Analog Scale (VAS) during the baseline menstrual period according to the patient-kept dairy. The main exclusion criteria were: 1) secondary dysmenorrhea related to endometriosis, endometrial polyps, pelvic inflammatory disease or uterine myoma; and 2) other 
gynecological problems. Patients enrolled in the acupuncture group were allowed to take NSAIDs at their usual usage and record the dosage in a painkiller diary which was collected.

In total, 83 patients were included in the acupuncture (high-dose: 12 acupoints: $\mathrm{N}=23$; low-dose: 6 acupoints, $\mathrm{N}=30$ ) and control ( $\mathrm{N}=30$ ) groups from 01, June, 2018 to 31, Dec. 2019. Patients were allowed to receive acupuncture or medication as their intention, yet those in the intervention groups were randomized to different doses (number of acupoints) of acupuncture by using random allocation software 2.0. Sequentially numbered containers were performed, all of the containers were tamper-proof, equal in weight, and similar in appearance. The study was performed in Traditional Medicine and Gynecological Departments, Taipei City Hospital from March 2019 to Dec. 2019. The trial was approved by the hospital Institutional/Independent Review Board (approval no. TCHIRB 10701111). The trial is registered at ClinicalTrials.gov, with number of NCT03881319. Figure 1 provides a flow chart of subject recruitment.

\section{Intervention}

In the acupuncture group, patients received acupuncture twice in each luteal phase, for three consecutive menstrual cycles, represented as Acupuncture 1-1; 1-

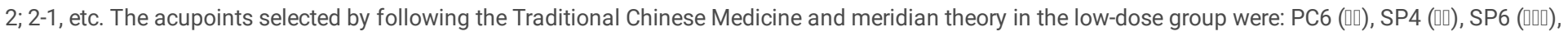

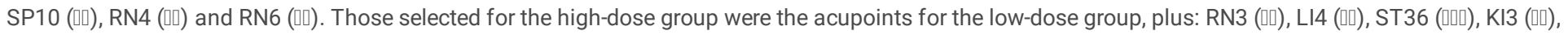
LR3(마) and EX-CA1 (ㅁ) (Figure 2). De-qi sensation(22) was manipulated with 20 mins of stimulation in each treatment session, using sterile disposable acupuncture needles. In the control (medication) group, participants took only NSAIDs with or without OCT. Patients in the acupuncture groups were allowed to take NSAIDs if they still felt pain and were asked to record the dosage used. The study included at least three consecutive menstrual cycles of treatment.

\section{Outcome measures}

To investigate the effect of acupuncture on autonomic function, we used HRV and analgesics dosage as the primary outcomes to assess the relationship and effect of different doses (number of acupoints stimulated) on autonomic nervous status and analgesic dependence. The HRV parameters included the time domain and frequency domain, before and after each course of intervention in both groups. To compare treatment efficacy by group, we used the VAS score to measure pain; the verbal multidimensional scoring system (VMSS), which asks participants to grade their ability to work, their systemic symptoms and their analgesics taking; and the 12-Item Short Form Health Survey (SF-12) quality of life questionnaire as secondary outcomes measurements.

\section{Statistical analysis}

In this trial, the estimated sample size was 34 in each group which determined by $\mathrm{G}$ power software, with $\mathrm{z}$ score:1.96, margin of error(e=0.1) , and the statistical analysis was performed using SPSS version 24.0 (IBM Corp., Armonk, NY). The data analysis was based on the intention-to-treat population. Means and standard deviations were determined for all data. We applied one-way ANOVA to compare the effect of acupuncture in the acupuncture and control groups, while the chi-squared test was used to analyze the categorical variables. The level of significance was established as $\mathrm{P}<=0.05 ; 95 \%$ confidence interval).

\section{Results}

In total, 98 patients were enrolled in the trial, 83 completed the trial: 23 in the high-dose group, 30 in the low-dose group and 30 in the control group. During the trial, no participants had any side effect from the intervention or treatment. Table 1 reveals no significant difference in baseline characteristics or HRV parameters between groups. 
Table 1

The characteristics of participants in the study

\begin{tabular}{|c|c|c|c|c|}
\hline & $\begin{array}{l}\text { Acupuncture high dose group }(\mathrm{N}= \\
\text { 23) }\end{array}$ & $\begin{array}{l}\text { Acupuncture low dose group }(\mathrm{N}= \\
30)\end{array}$ & $\begin{array}{l}\text { Control group }(\mathrm{N}= \\
30)\end{array}$ & $p_{\text {value }}$ \\
\hline & Mean (SD) & Mean (SD) & Mean (SD) & \\
\hline \multicolumn{5}{|l|}{ Basic data } \\
\hline Age(year) & $34.3(5.8)$ & $33.1(7.3)$ & $32.0(8.3)$ & .537 \\
\hline Body mass index $\left(\mathrm{kg} / \mathrm{m}^{2}\right)$ & $22.4(3.9)$ & $23.8(5.8)$ & $21.6(3.3)$ & .180 \\
\hline $\mathrm{SBP}(\mathrm{mmHg})$ & $72.0(3.6)$ & $71.5(5.1)$ & $71.8(6.7)$ & .907 \\
\hline $\mathrm{DBP}(\mathrm{mmHg})$ & $108.3(7.2)$ & 108.1(7.5) & $107.2(12.3)$ & .949 \\
\hline Heart rate(times/min) & $74.0(9.9)$ & $71.4(6.9)$ & $73.8(10.5)$ & .476 \\
\hline \multicolumn{5}{|l|}{ Menstruation related data } \\
\hline Age at menarche(years) & $12.4(1.3)$ & $12.9(1.6)$ & $12.4(1.7)$ & .343 \\
\hline Menstrual cycle duration(days) & $30.3(3.3)$ & $28.9(1.2)$ & $29.5(1.9)$ & .098 \\
\hline Menstrual irregularity, n(\%) & $1(4.3 \%)$ & $5(16.7 \%)$ & $4(13.3 \%)$ & .380 \\
\hline menstrual pain scores (VAS) & $5.3(1.5)$ & $5.9(1.6)$ & $5.6(1.8)$ & .335 \\
\hline \multicolumn{5}{|l|}{ Severity of dysmenorrhea (VMSS) } \\
\hline Grade $0-1$ : not painful or mild pain & $7(30.4 \%)$ & $4(13.3 \%)$ & $8(26.7 \%)$ & \multirow[t]{2}{*}{.281} \\
\hline Grade 2-3:moderate pain or severe pain & $16(69.6 \%)$ & $26(86.7 \%)$ & $22(73.3 \%)$ & \\
\hline \multicolumn{5}{|l|}{ Initial onset of menstrual pain, n(\%) } \\
\hline Menarche & $6(26.1 \%)$ & $10(33.3 \%)$ & $7(23.3 \%)$ & \multirow[t]{3}{*}{.160} \\
\hline$<3$ years after menarche & $1(4.3 \%)$ & $3(10.0 \%)$ & $8(26.7 \%)$ & \\
\hline Others & $16(69.6 \%)$ & $17(56.7 \%)$ & $15(50.0 \%)$ & \\
\hline $\begin{array}{l}\text { Use of medicine regulating menstruation, } \\
\mathrm{n}(\%)\end{array}$ & $5(21.7 \%)$ & $12(40.0 \%)$ & $9(30.0 \%)$ & .358 \\
\hline Painkillers (mg) & $173.9(387.6)$ & $741.7(1467.3)$ & 291.7(549.6) & .075 \\
\hline \multicolumn{5}{|l|}{ HRV-related parameters } \\
\hline $\operatorname{SDNN}(\mathrm{ms})$ & $43.0(15.9)$ & $46.9(15.7)$ & $45.8(27.8)$ & .785 \\
\hline R-MSSD(ms) & $36.6(16.5)$ & 41.5(17.3) & $43.7(26.4)$ & .465 \\
\hline PNN50(\%) & 18.7(17.3) & 23.0(19.2) & $21.5(21.5)$ & .727 \\
\hline $\operatorname{LF}(\%)$ & $53.1(16.0)$ & $48.9(19.8)$ & $57.3(14.8)$ & .169 \\
\hline LF (ms2) & $381.5(273.8)$ & $489.3(725.3)$ & $908.5(1555.4)$ & .145 \\
\hline HF (\%) & $46.9(16.0)$ & $51.1(19.8)$ & 42.7(14.8) & .169 \\
\hline $\mathrm{HF}(\mathrm{ms} 2)$ & $372.6(318.1)$ & $505.9(418.9)$ & $576.4(685.1)$ & .356 \\
\hline LF/HF & $1.44(1.00)$ & $1.44(1.44)$ & $1.84(1.98)$ & .543 \\
\hline VLF (ms2) & 1333.7(1193.8) & 1447.1(1071.6) & $1363.8(1703.0)$ & .950 \\
\hline $\mathrm{TP}(\mathrm{ms} 2)$ & 2087.8(1561.3) & 2442.3(1518.4) & 2848.6(3773.2) & .566 \\
\hline \multicolumn{5}{|l|}{ SF-12 quality of life scores } \\
\hline PCS & $42.4(8.6)$ & $41.5(7.6)$ & $42.2(6.9)$ & .904 \\
\hline MCS & $32.9(7.8)$ & $36.8(11.9)$ & $35.9(9.3)$ & .356 \\
\hline \multicolumn{5}{|l|}{ SBP: systolic blood pressure. } \\
\hline \multicolumn{5}{|l|}{ DBP: diastolic blood pressure. } \\
\hline \multicolumn{5}{|l|}{ VAS: visual analogue scale score. } \\
\hline \multicolumn{5}{|c|}{ VMSS: verbal multidimensional scoring system. } \\
\hline SDNN: standard deviation of adjacent $\mathrm{p}$ & $(\mathrm{NN})$ intervals & & & \\
\hline
\end{tabular}




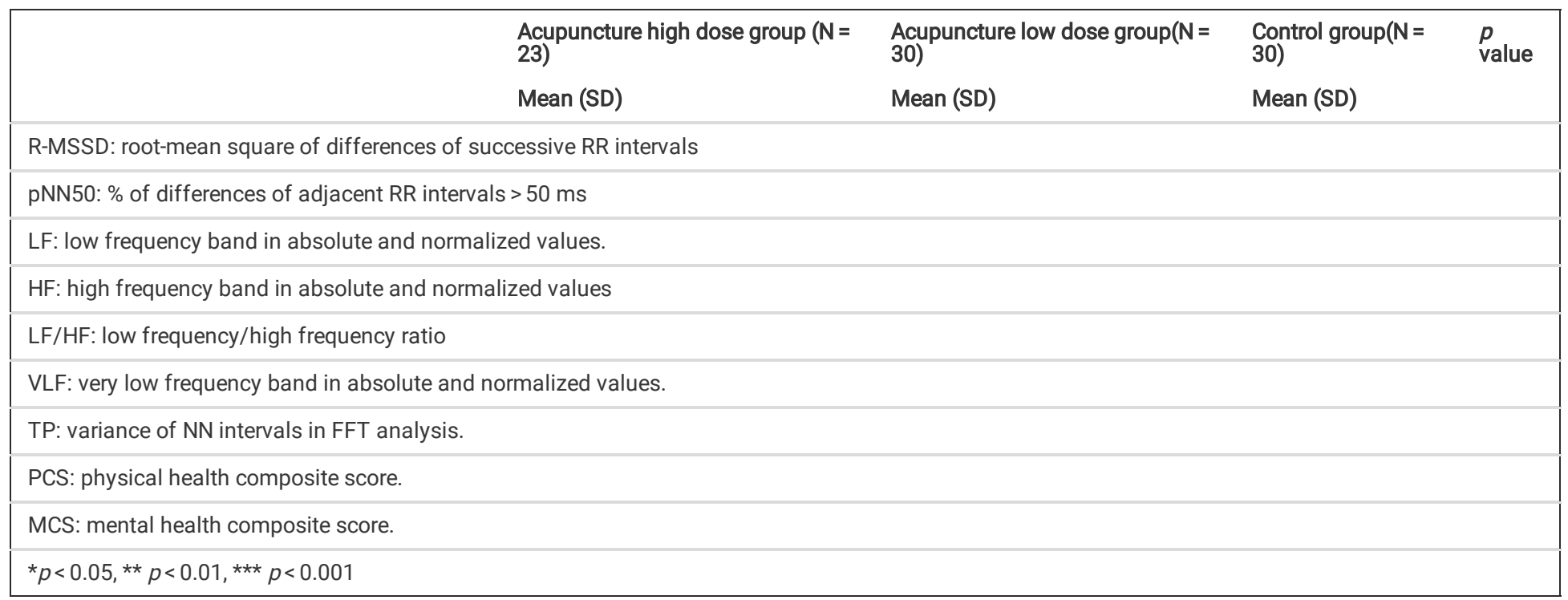

\section{The effect of acupuncture dosage on heart rate variation (HRV) parameters}

Table 2 compares the change in HRV parameters before and after treatment in the acupuncture and control groups. No significant difference was noted before and after the patients in control group who took 3 months of medication for their dysmenorrhea $(p>0.05)$. A similar phenomenon was noted in the acupuncture groups, with no significant change noted in patients' HRV parameters in the high-dose group, yet some meaningful improvement was found in the low-dose acupuncture group in both time and frequency domains (i.e., the percent of differences of adjacent RR intervals $>50 \mathrm{~ms}, \mathrm{p}=0.04$; the percentage of low frequency bands, $p=0.032$; and the percentage of high frequency bands, $p=0.032$ ). 
Table 2

The changes in autonomic nervous activity status before and after the intervention.

\begin{tabular}{|c|c|c|c|c|c|c|c|c|c|}
\hline & \multicolumn{3}{|c|}{ Acupuncture high dose group $(\mathrm{N}=23)$} & \multicolumn{3}{|c|}{ Acupuncture low dose group $(\mathrm{N}=30)$} & \multicolumn{3}{|c|}{ Control group $(\mathrm{N}=30)$} \\
\hline & Baseline & After 3 months & $p$ & Baseline & After 3 months & $p$ & Baseline & After 3 months & $p$ \\
\hline & Mean (SD) & Mean (SD) & & Mean (SD) & Mean (SD) & & Mean (SD) & Mean (SD) & \\
\hline \multicolumn{10}{|l|}{$\begin{array}{l}\text { Time } \\
\text { Domain }\end{array}$} \\
\hline SDNN & $43.0(15.9)$ & $41.0(14.0)$ & .580 & $46.9(15.7)$ & $46.8(15.7)$ & .974 & $45.8(27.8)$ & $48.0(17.0)$ & $.53 C$ \\
\hline $\begin{array}{l}\text { R- } \\
\text { MSSD (ms) }\end{array}$ & $36.6(16.5)$ & $35.2(17.2)$ & .711 & $41.5(17.3)$ & $36.4(14.0)$ & .054 & $43.7(26.4)$ & $45.9(22.1)$ & .56 \\
\hline PNN50(\%) & 18.7(17.3) & $15.4(15.6)$ & .382 & 23.0(19.2) & $16.4(14.3)$ & $.040 *$ & $21.5(21.5)$ & $22.1(18.2)$ & $.83 C$ \\
\hline \multicolumn{10}{|l|}{$\begin{array}{l}\text { Frequency } \\
\text { Domain }\end{array}$} \\
\hline LF (\%) & $53.1(16.0)$ & $50.7(17.3)$ & .474 & $48.9(19.8)$ & $55.8(19.3)$ & $.032^{\star}$ & $57.3(14.8)$ & $50.4(15.4)$ & $.05 \epsilon$ \\
\hline $\mathrm{LF}(\mathrm{ms} 2)$ & $381.5(273.8)$ & $394.5(395.7)$ & .868 & $489.3(725.3)$ & $503.1(426.0)$ & .929 & $908.5(1555.4)$ & $430.4(273.8)$ & .082 \\
\hline HF (\%) & $46.9(16.0)$ & 49.3(17.3) & .474 & 51.1(19.8) & $44.2(19.3)$ & $.032^{\star}$ & $42.7(14.8)$ & $49.6(15.4)$ & $.05 \epsilon$ \\
\hline $\mathrm{HF}(\mathrm{ms} 2)$ & $372.6(318.1)$ & $358.1(316.8)$ & .866 & $505.9(418.9)$ & $375.0(311.0)$ & .084 & $576.4(685.1)$ & $554.1(578.8)$ & $.82:$ \\
\hline LF/HF & $1.44(1.00)$ & $1.36(1.04)$ & .721 & $1.44(1.44)$ & $1.96(2.04)$ & .189 & 1.84(1.98) & $1.23(0.76)$ & $.10 ミ$ \\
\hline VLF (ms2) & 1333.7(1193.8) & 1115.3(681.5) & .356 & 1447.1(1071.6) & $1549.9(1475.3)$ & .778 & 1363.8(1703.0) & 1601.0(1099.5) & $.29 ?$ \\
\hline $\mathrm{TP}(\mathrm{ms} 2)$ & 2087.8(1561.3) & $1868.0(1127.2)$ & .513 & 2442.3(1518.4) & $2428.0(1820.6)$ & .975 & $2848.6(3773.2)$ & $2585.5(1827.1)$ & $.62 \varepsilon$ \\
\hline
\end{tabular}

SDNN: standard deviation of adjacent peak-to-peak (NN) intervals.

R-MSSD: root-mean square of differences of successive RR intervals.

pNN50: \% of differences of adjacent RR intervals $>50 \mathrm{~ms}$.

LF: low frequency band in absolute and normalized values.

HF: high frequency band in absolute and normalized values.

LF/HF: low frequency/high frequency ratio.

VLF: very low frequency band in absolute and normalized values.

TP: variance of NN intervals in FFT analysis.

${ }^{\star} p<0.05,{ }^{* *} p<0.01, * \star * p<0.001$

Despite the lack of obvious difference in between-group analysis, the intergroup-analysis of the acupuncture groups (Table 3) showed that patients had some improvement in autonomic nervous activity after certain acupuncture treatments. For example, in the high-dose acupuncture group, the Standard Deviation of Normal to Normal (SDNN, represents overall heart rate variability index) significantly changed after acupuncture session 1-1 (first menstrual cycle, first acupuncture session, $p=0.015)$; session $2-1(p=0.016)$; session $2-2(p=0.025)$; and session $3-1(p=0.031)$. HF (represent parasympathetic tone) significantly increased after sessions $2-2(p=0.042)$ and $3-1(p=0.025)$. Total power (TP), which represent autonomic activity, significant improved after sessions $1-1(p=0.013), 2-1(p=0.019)$ and $2-2(p=0.035)$. However, the significance of these changes was less in the low-dose acupuncture group. Nevertheless, the significant change distribution implied that acupuncture might have a short-term effect on some HRV parameters, but there was no obvious tendency of superiority for either high- or low-dose acupuncture (Table 3). 
Table 3

The comparison of short-term effect reflecting in each acupuncture session (intergroup- analysis) Valu

\begin{tabular}{|c|c|c|c|c|c|c|c|c|c|}
\hline & & $\begin{array}{l}\text { High dose } \\
(\mathrm{N}=23)\end{array}$ & $\begin{array}{l}\text { Low dose } \\
(\mathrm{N}=30)\end{array}$ & $\begin{array}{l}\text { High dose } \\
(\mathrm{N}=23)\end{array}$ & $\begin{array}{l}\text { Low dose } \\
(\mathrm{N}=30)\end{array}$ & $\begin{array}{l}\text { High dose } \\
(\mathrm{N}=23)\end{array}$ & $\begin{array}{l}\text { Low dose } \\
(\mathrm{N}=30)\end{array}$ & $\begin{array}{l}\text { High dose } \\
(\mathrm{N}=23)\end{array}$ & $\begin{array}{l}\text { Low dos } \\
(\mathrm{N}=30)\end{array}$ \\
\hline \multirow{3}{*}{$\begin{array}{l}\text { SDNN } \\
\text { (ms) }\end{array}$} & $B$ & $43.0(15.9)$ & $46.9(15.7)$ & $43.2(17.2)$ & $45.4(12.0)$ & $42.5(15.7)$ & 43.3(18.3) & 41.4(13.6) & $45.0(19.4$ \\
\hline & $A$ & $50.4(21.3)$ & $47.0(16.6)$ & 47.7(19.6) & 49.5(17.4) & $49.0(18.0)$ & $49.9(14.6)$ & $48.8(17.0)$ & 47.9(13.l \\
\hline & $p$ & $.015^{\star}$ & .976 & .199 & .121 & $.016^{*}$ & $.031 *$ & $.025^{\star}$ & .309 \\
\hline \multirow{3}{*}{$\begin{array}{l}\text { R- } \\
\text { MSSD } \\
(\mathrm{ms})\end{array}$} & B & $36.6(16.5)$ & $41.5(17.3)$ & $37.2(19.4)$ & $41.1(15.8)$ & $36.4(17.0)$ & $37.1(20.7)$ & $35.4(19.4)$ & 38.9(20.. \\
\hline & $A$ & $42.9(21.1)$ & $44.0(19.1)$ & $40.7(17.1)$ & $44.4(19.3)$ & $42.9(22.9)$ & $41.1(16.8)$ & $42.2(20.9)$ & 41.1(15.: \\
\hline & $p$ & $.010^{\star}$ & .304 & .219 & .153 & $.040 *$ & .226 & .087 & .479 \\
\hline \multirow{3}{*}{$\begin{array}{l}\text { PNN50 } \\
\text { (\%) }\end{array}$} & $\mathrm{B}$ & 18.7(17.3) & $23.0(19.2)$ & $17.8(19.6)$ & 22.7(17.9) & $16.2(14.1)$ & $18.3(20.8)$ & $15.4(18.6)$ & $19.8(20 .\{$ \\
\hline & $A$ & $25.1(20.8)$ & 24.4(19.3) & 21.8(17.7) & $25.2(20.0)$ & 23.3(21.3) & $22.7(17.8)$ & $22.1(20.2)$ & $22.0\left(18 .^{\circ}\right.$ \\
\hline & $p$ & $.008 * *$ & .581 & .219 & .304 & $.021^{\star}$ & .175 & .089 & .439 \\
\hline \multirow{3}{*}{$\begin{array}{l}\text { LF } \\
(\%)\end{array}$} & $B$ & $53.1(16.0)$ & $48.9(19.8)$ & $53.9(10.9)$ & $50.6(15.5)$ & $55.3(17.5)$ & $48.9(17.8)$ & $52.5(18.6)$ & 48.6(17.. \\
\hline & $A$ & $52.6(16.2)$ & $45.2(18.0)$ & $53.0(16.0)$ & $48.5(17.9)$ & $51.7(16.8)$ & $50.3(15.8)$ & $48.5(15.3)$ & $50.2(17$. \\
\hline & $p$ & .870 & .278 & .691 & .585 & .317 & .693 & .122 & .604 \\
\hline \multirow{3}{*}{$\begin{array}{l}\text { LF } \\
(\mathrm{ms} 2)\end{array}$} & B & $381.5(273.8)$ & 489.3(725.3) & $358.6(275.2)$ & $420.1(254.7)$ & $388.0(307.1)$ & $351.9(315.3)$ & 368.8(333.2) & $415.0(43$ \\
\hline & A & $559.8(550.1)$ & $502.5(513.9)$ & $520.2(500.4)$ & $589.4(550.5)$ & $527.2(430.0)$ & $528.6(437.6)$ & $504.7(523.4)$ & $558.8(72$ \\
\hline & $p$ & .069 & .932 & .097 & $.046^{*}$ & $.011 *$ & .050 & .165 & .318 \\
\hline \multirow{3}{*}{$\begin{array}{l}\mathrm{HF} \\
(\%)\end{array}$} & B & $46.9(16.0)$ & $51.1(19.8)$ & 46.1(10.9) & $49.4(15.5)$ & $44.7(17.5)$ & $51.1(17.8)$ & 47.5(18.6) & 51.4(17.. \\
\hline & A & $47.4(16.2)$ & $54.8(18.0)$ & $47.0(16.0)$ & $51.5(17.9)$ & $48.3(16.8)$ & $49.7(15.8)$ & $51.5(15.3)$ & 49.8(17.، \\
\hline & $p$ & .870 & .278 & .691 & .585 & .317 & .693 & .122 & .604 \\
\hline \multirow{3}{*}{$\begin{array}{l}\text { HF } \\
\text { (ms2) }\end{array}$} & $B$ & $372.6(318.1)$ & $505.9(418.9)$ & $362.0(373.2)$ & $461.0(347.7)$ & 381.3(393.4) & 413.7(442.9) & $389.7(421.0)$ & $447.0(46$ \\
\hline & A & $469.9(354.4)$ & $583.2(541.4)$ & 469.5(498.4) & $536.9(414.7)$ & 497.7(482.7) & $531.1(472.5)$ & $541.4(504.1)$ & $496.0(43$ \\
\hline & $p$ & .072 & .272 & .152 & .187 & .107 & .192 & $.042^{\star}$ & .593 \\
\hline \multirow[t]{3}{*}{ LF/HF } & B & $1.44(1.00)$ & $1.44(1.44)$ & $1.30(0.61)$ & $1.30(0.99)$ & $1.71(1.43)$ & $1.29(1.08)$ & $1.62(1.50)$ & $1.29\left(1.1^{\circ}\right.$ \\
\hline & $A$ & $1.48(1.30)$ & $1.21(1.35)$ & $1.53(1.39)$ & $1.34(1.38)$ & $1.34(0.97)$ & $1.33(1.13)$ & $1.17(0.86)$ & 1.51(1.7" \\
\hline & $p$ & .865 & .336 & .329 & .884 & .212 & .885 & .052 & .373 \\
\hline \multirow{3}{*}{$\begin{array}{l}\text { VLF } \\
\text { (ms2) }\end{array}$} & B & 1333.7(1193.8) & $1447.1(1071.6)$ & $1426.9(1111.9)$ & $1319.9(670.6)$ & 1271.9(912.2) & $1434.1(1373.0)$ & $1127.7(716.1)$ & $1524.2(1$ \\
\hline & $A$ & 1946.8(1955.1) & 1393.7(884.0) & 1648.4(1773.8) & $1618.8(1243.4)$ & $1681.9(1242.5)$ & 1636.1(1143.0) & 1613.7(1132.5) & $1403.3(8$ \\
\hline & $p$ & $.043^{*}$ & .828 & .462 & .187 & .104 & .302 & .068 & .572 \\
\hline \multirow{3}{*}{$\begin{array}{l}\text { TP } \\
\text { (ms2) }\end{array}$} & B & 2087.8(1561.3) & 2442.3(1518.4) & 2147.4(1578.7) & $2200.9(1037.6)$ & $2041.2(1472.5)$ & 2199.7(1939.0) & $1886.1(1257.7)$ & $2386.2(2$ \\
\hline & $A$ & 2976.5(2395.3) & $2479.4(1702.2)$ & 2638.1(2203.3) & 2745.1(1851.4) & $2706.8(1725.4)$ & 2695.8(1550.6) & 2659.9(1751.6) & $2458.1(1$ \\
\hline & $p$ & $.013^{*}$ & .915 & .190 & $.048^{*}$ & $.019 *$ & .114 & $.035^{\star}$ & .817 \\
\hline
\end{tabular}

B: before acupuncture.

A: after acupuncture.

SDNN: standard deviation of adjacent peak-to-peak (NN) intervals.

R-MSSD: root-mean square of differences of successive RR intervals.

pNN50: \% of differences of adjacent RR intervals $>50 \mathrm{~ms}$.

LF: low frequency band in absolute and normalized values.

HF: high frequency band in absolute and normalized values.

LF/HF: low frequency/high frequency ratio.

VLF: very low frequency band in absolute and normalized values.

TP: variance of NN intervals in FFT analysis.

Page $7 / 13$ 


\begin{tabular}{llllllll}
\hline High dose & Low dose & High dose & Low dose & High dose & Low dose & High dose & Low dos \\
$(\mathrm{N}=23)$ & $(\mathrm{N}=30)$ & $(\mathrm{N}=23)$ & $(\mathrm{N}=30)$ & $(\mathrm{N}=23)$ & $(\mathrm{N}=30)$ & $(\mathrm{N}=23)$ & $(\mathrm{N}=30)$
\end{tabular}

Acupuncture 1-1(A1-1): first week of 1st cycle.

Acupuncture 1-2(A1-2): second week of 1st cycle.

Acupuncture 2 - 1(A2-1): first week of 2nd cycle.

Acupuncture 2-2(A2-2): second week of 2nd cycle.

Acupuncture 3 -1(A3-1): first week of 3rd cycle.

Acupuncture 3 - 2(A3-2): second week of 3rd cycle.

${ }^{\star} p<0.05$, ** $p<0.01$, *** $p<0.001$

The effect of acupuncture dosage on reducing analgesics dependency and Quality of Life

As for use of painkillers, acupuncture could significantly reduce the level of painkiller or analgesic use after 3 months' treatment and low-dose acupuncture had a more significant benefit $(p=0.043)$ than high-dose acupuncture $(p=0.056)$. No significant change was noted in the control group $(p=0.376)$.

Furthermore, high-dose acupuncture $(p<0.001)$, low-dose acupuncture $(p<0.001)$ and medication $(p<0.001)$ all had a significant effect on relief of pain, as reflected in the VAS scores. The difference between the three groups not reach significance $(p=0.145)$. In terms of quality of life as measured by the SF-12, low-dose acupuncture improved participants' physical component score (PCS) much more meaningfully then did high-dose acupuncture (low-dose: $p<0.001$; high-dose: $p=0.001$; control: $p=0.007$ ). In the mental component score (MCS), high-dose acupuncture was superior to the other two groups, which implies a placebo effect (high-dose: $p<0.001$; low-dose: $p=0.025$; control: $p=0.01$ ) (Table 4).

Table 4

The improvement of analgesics dependence, pain (VAS), Quality of Life (SF12) and VMSS in acupuncture (high/low dose) and control group Acupuncture high dose group $(\mathrm{N}=23)$

Acupuncture low dose group $(\mathrm{N}=30)$

$\begin{array}{lll}\text { Baseline } & \text { After } 3 & p \\ \text { Mean (SD) } & \text { months } \\ & \text { Mean (SD) }\end{array}$

Painkillers $\quad 173.9(387.6) \quad 43.5(144.1) \dagger \quad .056$

(mg)

VAS $5.3(1.5)$

SF-12

PCS

MCS

42.4(8.6)

$49.5(6.4)$

$.001^{\star *}$

40.6(11.6)

$<$ $0.001^{\star * *}$

$32.9(7.8)$

$0.001^{\star \star *}$

Acupuncture high dose group $(\mathrm{N}=23)$

VMSS

Baseline $\begin{array}{ll}\text { After } 3 & p \\ \text { months } & \end{array}$

$\mathrm{N}(\%)$

$N(\%) \quad N(\%)$

Grade 0- $\quad 7(30.4 \%)$

1

Grade 2- $\quad$ 16(69.6\%) 5(21.7\%)

$18(78.3 \%) \quad .007^{\star \star}$

$p$

(2)

\begin{tabular}{lll}
$\begin{array}{ll}\text { Baseline } \\
\text { Mean (SD) }\end{array}$ & $\begin{array}{l}\text { After 3 } \\
\text { months } \\
\text { Mean (SD) }\end{array}$ & $p$ \\
\hline 741.7(1467.3) & $216.7(386.9)$ & .043
\end{tabular}

\section{Control group $(\mathrm{N}=30)$}

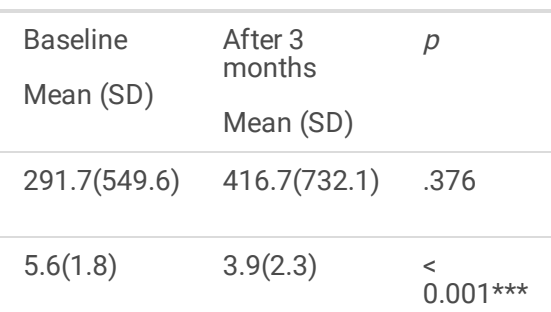

3

VAS: visual analogue scale score

QoL: quality of life

PCS: physical health composite score.

MCS: mental health composite score.

VMSS: Verbal multidimensional scoring system.

Grade $0-1$ : menstruation is not painful or mild pain.

Grade 2-3: Menstruation is moderate pain or severe pain.

${ }^{\star} p<0.05$, ** $p<0.01$, *** $p<0.001$ 
The effect of different doses of acupuncture on dysmenorrhea symptoms

In terms of severity of dysmenorrhea scores, patients in both acupuncture groups has significant improvement in VMSS scores. Low-dose acupuncture was more efficient in relieving symptoms than high-dose acupuncture (high-dose: $p=0.007$; low-dose: $p<0.001 ;$ control: $p=0.109$ ) (Table 4).

\section{Discussion}

\section{Dosage effect}

This is not the first study to confirm the efficacy of acupuncture in reliving primary dysmenorrhea(23, 24). However, few studies have evaluated the efficacy of different doses of acupuncture or the use of more acupoints. Acupuncture has been widely applied to diverse conditions: pain, arthritis, neuropathy, insomnia, and even some cancer-related side effects $(25,26)$. However, scholars and clinical physicians have not yet reached consensus on the optimal acupuncture prescription, including the number of acupoints, the number of acupuncture sessions, or even the location of acupoints or the optimal total dose. A study by Sun et al. (2019) pointed out that a higher dosage of acupuncture might provide better treatment outcomes in terms of relief of pain and dysfunction in patients with knee osteoarthritis(27), but others consider that more acupuncture sessions will only relieve symptoms in those with chronic pain(28). In primary dysmenorrhea, only one previous study has demonstrated that the timing of the acupuncture treatment and the mode of needle stimulation were germane to menstrual pain outcomes, but even this study failed to identify the optimum dose parameters for this condition(29). From our study, we noted that, when the parameters of acupuncture timing and frequency were fixed, acupuncture with fewer acupoints (lower dose) was not inferior to higher dose, and even better in terms of dependence on analgesics ( 6 acupoints: 0.043; 12 acupoints: 0.056$)$; dysmenorrhea symptom control (VMSS: 6 acupoints: $p<0.001 ; 12$ acupoints: $p=0.007$ ); and quality of life (SF-12 PCS: 6 acupoints: $p<0.001 ; 12$ acupoints: $p=0.001$ ). Therefore, the clinical physician should understand that clinical efficacy is not depend on the number of acupoints and should convey this concept to patients, to reduce the waste of medical resources(9). In quality of life assessment, we found that subjects had more improvement in mental quality of life than in physiological quality of life, so that the placebo effect should be considered a relevant psychological factor. In this regard, a study claimed that the correlation coefficients between de-qi and the therapeutic efficacy of acupuncture were greater than those between psychological factors and therapeutic efficacy in dysmenorrhea(30). Therefore, the mechanisms driving acupuncture efficacy are complex and extend beyond the simplistic "more is better" concept of treatment.

\section{Relationship to autonomic activity adjustment}

Some studies indicate that de-qi is the most important factor in making acupuncture effective(31). A study in 2017 revealed that patients with dysmenorrhea who experienced actual de-qi sensation had more pain relief and a greater analgesic effect(32). An interesting study revealed that de-qi elicited a significant response to acupuncture in specific brain regions, indicating that de-qi acupuncture triggers the neuroendocrine network to alleviate pain, but the mechanisms are not clear(22). In our study, we used HRV as an outcome measure, to check whether the acupuncture effect was correlated to autonomic change. HRV has long been recognized as a noninvasive indicator of autonomic nervous system activity $(20,33)$. Indeed, HRV coherence is related to many physiological benefits, including short-term improvement in cardiovascular and respiratory function, and an increase in vagal afferent activity, which converses pain signals and sympathetic outflow, and thereby promotes temporal synchronization of the body $(34,35)$. Accordingly, the improvement in autonomic dysfunction corresponds with the balance of yin/yang and implies the adjustment of neuroendocrine in the body microenvironment(20). In this study, we noted that acupuncture has only a short-term effect in adjusting the autonomic dysfunction within the acupuncture group; the benefit did not meaningfully outweigh that received by the medication (control) group ( $p>0.05$ ). Nevertheless, inter-group analysis revealed that subjects in the high-dose group had greater HRV change, a result which can be interpreted two ways. First, the effect of acupuncture on the autonomic system is short-term rather than long-term. Second, the stimulation of more acupoints may lead to more change in HRV activity, but not greater efficacy in pain relief or symptom control. The mechanism by which acupuncture relieves dysmenorrhea may not directly derive from a change in autonomic nervous activity. We therefore wonder whether the effect of acupuncture in dysmenorrhea is correlated with alterations in serum cytokines, such prostaglandin E and prostaglandin F2 alpha, as has been revealed in previous literature(36). Further study to evaluate the role of serum cytokines is encouraged.

\section{Potential mechanisms of pain reduction}

The mechanism of pain associated with dysmenorrhea can be addressed in two aspects: the neuro-endocrine regulated and the neuro-physiologic related. As our study indicates that subjects who adopt acupuncture could use less analgesics, we suppose that acupuncture may have some neuro-endocrine effect(37, 38). In this regard, a previous study noted that acupuncture could have both an anti-inflammatory and an analgesic effect, which might be associated with its inhibition of spinal p38MAPK activation(39) or its regulation of serum prostaglandin E2 or F2 alpha(40). Because patients who used acupuncture required less NSAIDs, acupuncture may have a similar anti-inflammatory effect as NSAIDs, but without the side effects of gastrointestinal discomfort or ulcer stimulation.

On the other hand, some studies have noted that the shift in the estradiol/progesterone ratio in favor of estradiol seems to be the pathogenic principle behind dysmenorrhea $(41,42)$. We therefore suppose that acupuncture might somehow affect the related hormone balance. In animal models, acupuncture had been noted to regulate neuroendocrine activity and the related receptor expression of the hypothalamus-pituitary-ovary (HPO) axis(38, 43). Clinically, some gynecologist also use oral contraceptives or progestins (OCT) for primary dysmenorrhea(44). If acupuncture could adjust the HPO axis and thereby relieve the related symptoms, then patients can avoid taking OCTs which might have unwanted side effects such as increased risk of endometriosis, blood clot, stroke or cancer(45).

\section{Holistic effect of acupuncture on dysmenorrhea}


The results indicate that acupuncture could reduce subjects' dependence on analgesics and improve their VAS and VMSS scores, which means increasing their ability to work and allowing them to resume more normal activity with fewer symptoms. Compared to the alternatives, acupuncture has fewer side effects and is more cost-effective, especially considering that the number of acupoints is not linearly related to outcomes.

\section{Limitations}

We noted some bias and limitations regarding the study. The first is that the size of the study could me more to be more powerful. Second, there is as yet no consensus on the optimal acupuncture schedule for any individual disease. We designed the schedule based on the Traditional Chinese Medicine meridian theory, but others might select different acupoints and devise a different schedule when treating the same condition.

\section{Conclusions}

We conclude that acupuncture can mitigate dysmenorrhea in terms of providing pain relief, reducing analgesic dependence, improving symptoms and improving quality of life. Furthermore, treatment efficacy is not directly proportional to the dose or number of acupoints stimulated. A well-designed acupuncture schedule with de-qi maneuver is probably more important than the number of needles. We thus concluded that the effect of acupuncture in dysmenorrhea is not direct correlated with the autonomic nervous system adjustment. Acupuncture might have a short-term effect in changing the HRV parameters, yet the true mechanism for acupuncture in dysmenorrhea still warrants further investigation.

\section{Abbreviations}

\section{SF-12}

12-Item Short Form Health Survey; HRV:Heart rate variation; HF:High frequency; HPO:Hypothalamus-pituitary-ovary; LF:Low frequency; NSAIDs:Non-Steroidal Anti-Inflammatory Drugs; OCT:Oral contraceptive pills; R-MSSD:root-mean square of differences of successive RR intervals; SDNN:standard deviation of adjacent peak-to-peak (NN) intervals; TP:Total power; VMSS:Verbal Multidimensional Scoring System; VAS:Visual Analog Scale; VLF:Very low frequency.

\section{Declarations}

\section{Ethics approval and consent to participate}

Not applicable

\section{Consent for publication}

Not applicable.

\section{Availability of data and materials}

The datasets used for the current study are available upon reasonable request from the corresponding author.

\section{Competing Interests}

The authors declare that they have no competing interests.

\section{Funding}

The study is supported by Ministry of Science and Technology - MOST, Taiwan grant 107-2635-B-532-003- and grant 108-2320-B-532 -001 -MY3

\section{Author's contributions}

1. Tsai-Ju Chien*, MD.MA.MS.PhD: Study Design and conception; Drafting of the manuscript

2. Yi-Shuo Huang MD: Acquisition of data

3. Chun-Yu Kuo MS; Analysis and interpretation of data; statistical analysis

4. Yu Ching Cho MD: Acquisition of data

5. Hsin Yu Chen MD: Acquisition of data

6. Chi-Chang Chu MD: Acquisition of data

7. Ting-Yu Cheng MS: literature research

\section{Acknowledgement}

We thank all authors and participants in this study

\section{References}

1. Bernardi M, Lazzeri L, Perelli F, Reis FM, Petraglia F. Dysmenorrhea and related disorders. F1000Res. 2017;6:1645. 
2. De Sanctis V, Soliman AT, Elsedfy H, Soliman NA, Soliman R, El Kholy M. Dysmenorrhea in adolescents and young adults: a review in different country. Acta Biomed. 2017;87(3):233-46.

3. Harel Z. Dysmenorrhea in adolescents and young adults: etiology and management. J Pediatr Adolesc Gynecol. 2006;19(6):363-71.

4. Harel Z. Dysmenorrhea in adolescents and young adults: an update on pharmacological treatments and management strategies. Expert Opin Pharmacother. 2012;13(15):2157-70.

5. Alsaleem MA. Dysmenorrhea, associated symptoms, and management among students at King Khalid University, Saudi Arabia: An exploratory study. J Family Med Prim Care. 2018;7(4):769-74.

6. Smith CA, Zhu X, He L, Song J. Acupuncture for primary dysmenorrhoea. Cochrane Database Syst Rev. 2011(1):CD007854.

7. Sun YN, Hou XS, Wu JY, Tian HF, Zhao JP. [Acupuncture Treatment of Primary Dysmenorrhea by Needling Acupoints of the Spleen Meridian with Positive ReactionsA Randomized Controlled Clinical Trial]. Zhen Ci Yan Jiu. 2018;43(5):307-10.

8. Sriprasert I, Suerungruang S, Athilarp P, Matanasarawoot A, Teekachunhatean S. Efficacy of Acupuncture versus Combined Oral Contraceptive Pill in Treatment of Moderate-to-Severe Dysmenorrhea: A Randomized Controlled Trial. Evid Based Complement Alternat Med. 2015;2015:735690.

9. Schwehr NA, Shippee ND, Johnson PJ. Acupuncture 'dose' (number of treatments) and insurance benefits in the USA. Acupunct Med. 2018;36(2):88-95.

10. Hasegawa N. [Dysmenorrhea and the autonomic nervous system]. Sanfujinka No Jissai. 1968;17(6):492-8.

11. Oladosu FA, Hellman KM, Ham PJ, Kochlefl LE, Datta A, Garrison EF, et al. Persistent autonomic dysfunction and bladder sensitivity in primary dysmenorrhea. Sci Rep. 2019;9(1):2194.

12. Wang YJ, Wang YZ, Yeh ML. A Prospective Comparison Study of Heart Rate Variability During Menses in Young Women With Dysmenorrhea. Biol Res Nurs. 2016;18(4):465-72.

13. Shankar N, Thakur M, Tandon OP, Saxena AK, Arora S, Bhattacharya N. Autonomic status and pain profile in patients of chronic low back pain and following electro acupuncture therapy: a randomized control trial. Indian J Physiol Pharmacol. 2011;55(1):25-36.

14. Shu Q, Wang H, Litscher D, Wu S, Chen L, Gaischek I, et al. Acupuncture and Moxibustion have Different Effects on Fatigue by Regulating the Autonomic Nervous System: A Pilot Controlled Clinical Trial. Sci Rep. 2016;6:37846.

15. Liu T, Yu JN, Cao BY, Peng YY, Chen YP, Zhang L. Acupuncture for Primary Dysmenorrhea: A Meta-analysis of Randomized Controlled Trials. Altern Ther Health Med. 2017.

16. Chang S. The meridian system and mechanism of acupuncture: a comparative review. Part 3: Mechanisms of acupuncture therapies. Taiwan J Obstet Gynecol. 2013;52(2):171-84.

17. Yang W. Investigation of the lower resistance meridian: speculation on the pathophysiological functions of acupuncture meridians. Evid Based Complement Alternat Med. 2014;2014:107571.

18. Wu DZ. Acupuncture and neurophysiology. Clin Neurol Neurosurg. 1990;92(1):13-25.

19. Joshi N, Araque H. Neurophysiologic basis for the relief of human pain by acupuncture. Acupunct Electrother Res. 2009;34(3-4):165-74.

20. Chien TJ, Liu CY, Lu RH, Kuo CW, Lin YC, Hsu CH. Therapeutic efficacy of Traditional Chinese medicine, "Kuan-Sin-Yin", in patients undergoing chemotherapy for advanced colon cancer - A controlled trial. Complement Ther Med. 2016;29:204-12.

21. Lefebvre G, Pinsonneault O, Antao V, Black A, Burnett M, Feldman K, et al. Primary dysmenorrhea consensus guideline. J Obstet Gynaecol Can. 2005;27(12):1117-46.

22. Tian DS, Xiong J, Pan Q, Liu F, Wang L, Xu SB, et al. De qi, a threshold of the stimulus intensity, elicits the specific response of acupoints and intrinsic change of human brain to acupuncture. Evid Based Complement Alternat Med. 2014;2014:914878.

23. Witt CM, Reinhold T, Brinkhaus B, Roll S, Jena S, Willich SN. Acupuncture in patients with dysmenorrhea: a randomized study on clinical effectiveness and cost-effectiveness in usual care. Am J Obstet Gynecol. 2008;198(2):166 e1-8.

24. Luo F, Huang X, Liu X, Wang L, Xu N. Comparative efficacy and safety of NSAIDs-controlled acupuncture in the treatment of patients with primary dysmenorrhoea: a Bayesian network meta-analysis. J Int Med Res. 2019;47(1):19-30.

25. Chien TJ, Liu CY, Fang CJ. The Effect of Acupuncture in Breast Cancer-Related Lymphoedema (BCRL): A Systematic Review and Meta-Analysis. Integr Cancer Ther. 2019;18:1534735419866910.

26. Chien TJ, Liu CY, Fang CJ, Kuo CY. The maintenance effect of acupuncture on breast cancer-related menopause symptoms: a systematic review. Climacteric. 2019:1-10.

27. Sun N, Tu JF, Lin LL, Li YT, Yang JW, Shi GX, et al. Correlation between acupuncture dose and effectiveness in the treatment of knee osteoarthritis: a systematic review. Acupunct Med. 2019;37(5):261-7.

28. Qin Z, Wu J, Xu C, Liu Z. Using meta-regression approach to explore the dose-response association between acupuncture sessions and acupuncture effects on chronic prostatitis/chronic pelvic pain syndrome. Ann Transl Med. 2019;7(6):116.

29. Armour M, Smith CA. Treating primary dysmenorrhoea with acupuncture: a narrative review of the relationship between acupuncture 'dose' and menstrual pain outcomes. Acupunct Med. 2016;34(6):416-24.

30. Xiong J, Liu F, Zhang MM, Wang W, Huang GY. De-qi, not psychological factors, determines the therapeutic efficacy of acupuncture treatment for primary dysmenorrhea. Chin J Integr Med. 2012;18(1):7-15.

31. Kong J, Gollub R, Huang T, Polich G, Napadow V, Hui K, et al. Acupuncture de qi, from qualitative history to quantitative measurement. J Altern Complement Med. 2007;13(10):1059-70. 
32. Zhao MY, Zhang P, Li J, Wang LP, Zhou W, Wang YX, et al. Influence of de qi on the immediate analgesic effect of SP6 acupuncture in patients with primary dysmenorrhoea and cold and dampness stagnation: a multicentre randomised controlled trial. Acupunct Med. 2017;35(5):332-8.

33. Uchida C, Waki H, Minakawa Y, Tamai H, Miyazaki S, Hisajima T, et al. Effects of Acupuncture Sensations on Transient Heart Rate Reduction and Autonomic Nervous System Function During Acupuncture Stimulation. Med Acupunct. 2019;31(3):176-84.

34. Evans S, Seidman LC, Tsao JC, Lung KC, Zeltzer LK, Naliboff BD. Heart rate variability as a biomarker for autonomic nervous system response differences between children with chronic pain and healthy control children. J Pain Res. 2013;6:449-57.

35. Napadow V, Lee J, Kim J, Cina S, Maeda Y, Barbieri R, et al. Brain correlates of phasic autonomic response to acupuncture stimulation: an event-related fMRI study. Hum Brain Mapp. 2013;34(10):2592-606.

36. Sun L, Liang Y, Li X, Liu L, Xu X, Ma H, et al. [Efficacy of acupuncture combined with auricular point sticking on the content of serum prostaglandin F2alpha, and plasma arginine vasopressin in patients with menstrual headache]. Zhongguo Zhen Jiu. 2015;35(2):137-40.

37. Yu JS, Zeng BY, Hsieh CL. Acupuncture stimulation and neuroendocrine regulation. Int Rev Neurobiol. 2013;111:125-40.

38. Liu F, Xiong J, Huang GY, Wang W. [Study on the underlying mechanism of acupuncture in regulating neuroendocrine activity in dysmenorrhea rats]. Zhen Ci Yan Jiu. 2009;34(1):3-8.

39. Liang F, Chen R, Cooper EL. Neuroendocrine mechanisms of acupuncture. Evid Based Complement Alternat Med. 2012;2012:792793.

40. Hu YL, Lin Q, Li Y, Zheng XH. [Effect on PGF2alpha in plasma in primary dysmenorrhea treated with eye acupuncture]. Zhongguo Zhen Jiu. 2011;31(8):683-6.

41. Zahradnik HP, Breckwoldt M. Contribution to the pathogenesis of dysmenorrhea. Arch Gynecol. 1984;236(2):99-108.

42. Zhu H, Nan S, Suo C, Zhang Q, Hu M, Chen R, et al. Electro-Acupuncture Affects the Activity of the Hypothalamic-Pituitary-Ovary Axis in Female Rats. Front Physiol. 2019;10:466.

43. Feng Y, Johansson J, Shao R, Manneras L, Fernandez-Rodriguez J, Billig H, et al. Hypothalamic neuroendocrine functions in rats with dihydrotestosteroneinduced polycystic ovary syndrome: effects of low-frequency electro-acupuncture. PLoS One. 2009;4(8):e6638.

44. Oral contraceptives are effective for dysmenorrhea. J Fam Pract. 2005;54(10):843.

45. Chapron C, Souza C, Borghese B, Lafay-Pillet MC, Santulli P, Bijaoui G, et al. Oral contraceptives and endometriosis: the past use of oral contraceptives for treating severe primary dysmenorrhea is associated with endometriosis, especially deep infiltrating endometriosis. Hum Reprod. 2011;26(8):2028-35.

\section{Figures}

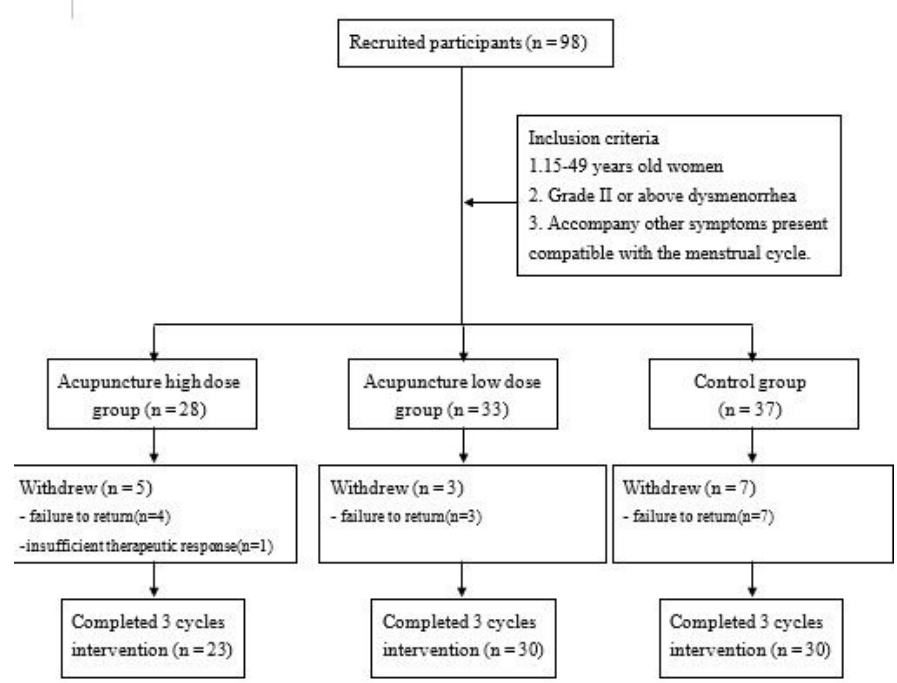

\section{Figure 1}

Flow chart of study 


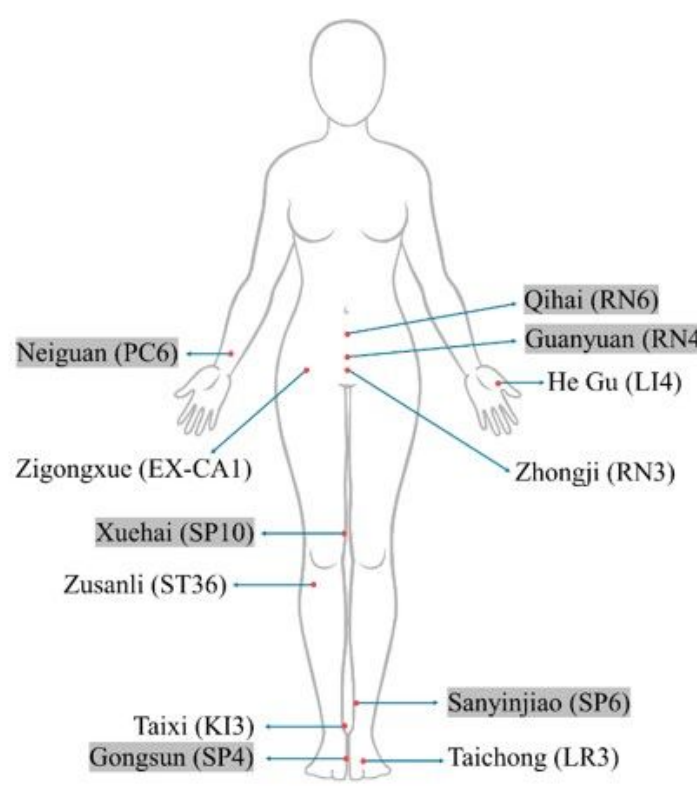

Figure 2

Selected acupoints in the study high dose acupuncture group: All acupoints low dose acupuncture group囚gray-colored mark: PC6, SP4, SP6, SP10, RN4,RN6

\section{Supplementary Files}

This is a list of supplementary files associated with this preprint. Click to download.

- CONSORT2010Checklist.doc

- CONSORT2010Checklist.doc

\begin{tabular}{|c|c|}
\hline RN6 & $\begin{array}{l}\text { On the anterior median line of the lower abdomen, } \\
1.5 \text { cun below the umbilicus. }\end{array}$ \\
\hline RN4 & $\begin{array}{l}3 \text {-cun below the umbilicus, on the midline of the - } \\
\text { abdomen. }\end{array}$ \\
\hline SP4 & $\begin{array}{l}\text { On the medial aspect of the foot, in the depression. } \\
\text { distal and 'inferior to the base 'of the first metatarsal- } \\
\text { bone. }\end{array}$ \\
\hline SP6 & $\begin{array}{l}\text { On the medial aspect 'of the 'lower leg, } 3 \text { 'cun above- } \\
\text { the medial malleolus, on the posterior border of ' } \\
\text { the medial aspect of the tibia. }\end{array}$ \\
\hline SP10 & 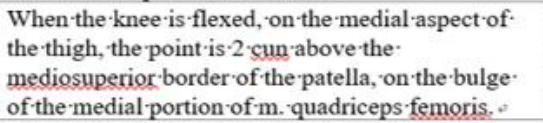 \\
\hline PC6. & $\begin{array}{l}\text { On the palmar 'side of the wrist two inches above' } \\
\text { the transverse'crease 'of the wrist, between the- } \\
\text { tendons 'of palmaris'longus and flexor'carpi- } \\
\text { radialis. }\end{array}$ \\
\hline LI4 & $\begin{array}{l}\text { On the highest 'spot of the muscle when the thumb. } \\
\text { and 'index fingers are brought 'close together. }\end{array}$ \\
\hline RN3 & 4-cun below the umbilicus on the mid-line. \\
\hline LR3 & $\begin{array}{l}\text { Just distal to the junction of the bases 'of the - Ist' } \\
\text { and } 2 \text { nd metatarsals. }\end{array}$ \\
\hline KI3. & $\begin{array}{l}\text { On the medial aspect of the foot, posterior to the } \\
\text { medial 'malleolus, 'in the depression between the tip } \\
\text { of the medial malleolus and tendo'calcaneus. }\end{array}$ \\
\hline ST36。 & $\begin{array}{l}\text { On the anterior aspect of the - lower-leg, one finger- } \\
\text { breadth-(middle finger) from the anterior 'crest 'of- } \\
\text { the tibia. }\end{array}$ \\
\hline EX-CA1 & $\begin{array}{l}3 \text {-cun-lateral to the anterior midline and } 1 \text {-cun- } \\
\text { superior to the upper border of the pubic - } \\
\text { symphysis. }\end{array}$ \\
\hline
\end{tabular}

